\section{(A) OPEN ACCESS}

\title{
High-sensitivity troponin assay improves prediction of cardiovascular risk in patients with cerebral ischaemia
}

\author{
Raoul Stahrenberg, ${ }^{1}$ Cord-Friedrich Niehaus, ${ }^{1}$ Frank Edelmann, ${ }^{1}$ Meinhard Mende, ${ }^{2}$ \\ Janin Wohlfahrt, ${ }^{3}$ Katrin Wasser, ${ }_{1}^{3}$ Joachim Seegers, ${ }^{1}$ Gerd Hasenfuß, ${ }^{1}$ \\ Klaus Gröschel, ${ }^{3,4}$ Rolf Wachter ${ }^{1}$
}

\begin{abstract}
${ }^{1}$ Department of Cardiology and Pneumology, University of Göttingen, Göttingen, Germany ${ }^{2}$ Centre for Clinical Trials, University of Leipzig, Leipzig, Germany

${ }^{3}$ Department of Neurology, University of Göttingen, Göttingen, Germany

${ }^{4}$ Department of Neurology, University of Mainz, Mainz, Germany
\end{abstract}

\section{Correspondence to} Dr Raoul Stahrenberg, Department of Cardiology and Pneumology, University of Göttingen, Robert-Koch-Str. 40, Göttingen 37075, Germany;

stahrenberg@med.unigoettingen.de

KG and RW contributed equally.

Received 1 June 2012 Revised 10 December 2012 Accepted 18 December 2012 Published Online First 25 January 2013

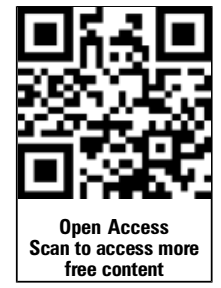

To cite: Stahrenberg $R$, Niehaus $C-F$, Edelmann $F$ et al. I Neurol Neurosurg Psychiatry 2013:84: 479-487.

\section{ABSTRACT}

Background and purpose Clinical scores are recommended for predicting cardiovascular risk in patients with cerebral ischaemia to inform secondary prevention. Blood biomarkers may improve prediction beyond clinical scores.

Methods Within the observational Find-AF trial (ISRCTN46104198), 197 patients $>18$ years of age with cerebral ischaemia and without atrial fibrillation had blood sampled at baseline. The predictive value of five biomarkers for a combined vascular endpoint (acute coronary syndrome, stroke, cardiovascular death) and all-cause mortality was determined, alone and in addition to the Essen Stroke Risk Score (ESRS), Stroke Prognostic Instrument 2 (SPI-2) and National Institutes of Health Stroke Scale (NIH-SS).

Results There were 23 vascular events (11.7\%) and 13 deaths $(6.6 \%)$ to 1 year follow-up. In multivariate analyses of all markers, only high-sensitivity troponin $\mathrm{T}$ (hsTropT) remained independently predictive for vascular events ( $p=0.045)$ and all-cause mortality $(p=0.004)$. hsTropT was higher in patients with a vascular event (median $12.7 \mathrm{ng} / \mathrm{ml}$ vs $5.1 \mathrm{ng} / \mathrm{ml}$ ), and patients with hsTropT above the median of $6.15 \mathrm{ng} / \mathrm{ml}$ had vascular events more frequently (HR 3.86, $p=0.008)$. For prediction of vascular events as well as all-cause mortality, hsTropT significantly improved multivariate Cox regression models with ESRS, SPI-2 or $\mathrm{NIH}-\mathrm{SS}$. The c-statistic increased non-significantly from 0.695 (ESRS) or 0.710 (hsTropT) to 0.747 (ESRS +hsTropT) and from 0.699 (SPI-2) to 0.763 (SPI-2 +hsTropT). No patient with a low-risk ESRS and an hsTropT below the median had a vascular event or died. Conclusions hsTropT predicts vascular events and allcause mortality in patients with acute cerebral ischaemia and improves prediction beyond established clinical scores.

\section{INTRODUCTION}

Patients who have experienced cerebral ischaemic events are at high risk for recurrent ischaemia ${ }^{1}$ and other cardiovascular events. ${ }^{2} 3$ Identifying those at highest risk may improve individualised secondary preventive measures. Clinical risk scores like the Essen Stroke Risk Score (ESRS) or Stroke Prognostic Instrument 2 (SPI-2) have been developed $^{4-6}$ and validated ${ }^{7-11}$ for this purpose. They are valuable tools for prognosticating future risk to patients and families and for risk stratification for clinical trials. Differential recommendations for secondary prevention have been based on these scores. ${ }^{5}$ 12 Although these tools are therefore clinically useful, their predictive value is moderate ${ }^{11}$ with room for further improvement.

Plasma biomarkers have been widely investigated for diagnostic and prognostic purposes in cardiovascular patient populations. A number of markers have been investigated in stroke patients ${ }^{13}$ and several were found to be predictive of recurrent vascular events including stroke. Markers of inflammation such as C-reactive protein (CRP) or IL-6 and markers of procoagulation such as D-dimer or fibrinogen, ${ }^{14} 15$ however, are not specific for vascular disease and none of these was deemed predictive enough to be used in clinical practice recommendations. More recently introduced markers offer potential technical advantages such as the measurement of biologically more stable precursor peptides or assays with higher sensitivity, and the combination of several markers in a multimarker approach may possibly improve prognostication. ${ }^{16-18}$

We investigated the value of five novel cardiovascular biomarkers in comparison with and in addition to established clinical risk scores to predict adverse cardiovascular outcomes in an unselected cohort of patients with cerebral ischaemia in the observational Find-AF trial.

\section{METHODS}

Patients

Find-AF was a monocentric prospective observational trial (ISRCTN46104198) that recruited consecutive patients presenting to the emergency department of University Hospital Göttingen between March 2009 and February 2010 with symptoms of stroke or transient ischaemic attack (TIA). The primary objective of Find-AF was the identification of factors associated with paroxysmal atrial fibrillation to guide diagnosis and therapy. Details of the study design have been published previously. ${ }^{1920}$ For the present analysis, all patients were included who did not have documented atrial fibrillation (by history, admission ECG or on 7-day Holter monitoring) and completed follow-up.

Find-AF complies with the Declaration of Helsinki, the protocol was approved by the 
responsible ethics committee at the University of Göttingen, and all patients gave written informed consent.

\section{Biomarkers}

Blood was drawn from patients as early as possible on presentation to the emergency department, immediately centrifuged and stored at $-80^{\circ} \mathrm{C}$ for later analysis. A set of five biomarkers was selected that have been shown to be prognostic for cardiovascular events in populations with stroke or other manifestations of atherosclerosis, mostly acute coronary syndromes (ACS). Three of these are indicative of cardiac haemodynamic stress (N-terminal pro brain natriuretic peptide (NT-proBNP), ${ }^{21} 22$ $\mathrm{N}$-terminal pro atrial natriuretic peptide (NT-proANP) $)^{23} 24$ and growth differentiation factor 15 (GDF-15) ${ }^{17} 25$ ), while the other two are released by myocardial necrosis (high-sensitivity tropo$\operatorname{nin} \mathrm{T}$ (hsTropT) ${ }^{26} 27$ and heart-type fatty acid binding protein $\left.(\mathrm{h}-\mathrm{FABP})^{28}\right)$. All markers were measured in EDTA-plasma: GDF-15, hsTropT and NT-proBNP by an electrochemiluminescence assay on an Elecsys analyser (Roche Diagnostics, Mannheim, Germany); BNP by sandwich-immunoluminescence assay on a Centaur analyser (Bayer Vital, Leverkusen, Germany); and proANP manually by an enzyme-immuno assay (Biomedica, Vienna, Austria). Except for GDF-15, ${ }^{29}$ all assays are commercially available. Measurements were kindly performed at Roche Diagnostics, Mannheim, Germany, blinded to clinical and outcome data.

\section{Study procedures and endpoints}

Clinical characteristics were recorded using a standardised questionnaire. Comorbidities relevant for this analysis were assessed on presentation and as judged by the enrolling physician (eg, arterial hypertension when the patient reported a history of hypertension or were prescribed antihypertensives such as ACE inhibitors with no alternative indication). All patients received a carotid ultrasound and cerebral scan (CT or MRI) and were treated in our certified stroke unit for at least $24 \mathrm{~h}$. As previously described, paroxysmal atrial fibrillation was diagnosed by 7-day Holter monitoring (CardioMem CM 3000, GETEMED Medizin- und Informationstechnik, Teltow, Germany). ${ }^{19}$

Patients were followed up after 3 months by telephone and after 12 months in a clinical visit. Patients unable to attend a clinical visit were either visited at home or followed up by telephone for endpoints, presence of atrial fibrillation, Modified Rankin Scale and current medication. For patients unable to answer themselves first degree relatives or their primary care physician were questioned.

Clinical endpoints that were prospectively assessed in the case report form in Find-AF were the occurrence of stroke, TIA, ACS, coronary revascularisation by bypass grafting or percutaneous intervention, revascularisation of peripheral arteries, cerebrovascular revascularisation, hospitalisation for heart failure, hospitalisation for other causes and death. Aetiology of death was adjudicated by the authors, blinded to biomarker values, to be cardiovascular or non-cardiovascular. For all endpoints reported, original documents were obtained from primary care providers or treating hospitals for validation.

\section{Definitions and statistical analyses}

The present analysis was not prespecified and was performed post hoc. A combined cardiovascular endpoint of stroke, ACS or cardiovascular death was constructed (referred to as 'vascular events' throughout this manuscript), because such an endpoint has previously been used for the development of cardiovascular risk scores, and because its components are representative of the same pathophysiological domain (triggering similar secondary preventive measures) and could be considered to be of approximately similar importance from a patient's perspective with regard to decisions concerning secondary preventive measures, for example, aggressive lifestyle modification.

ESRS and SPI-2 were retrospectively calculated from the individual components prospectively assessed on presentation. ESRS and SPI-2 have been shown to have predictive power similar to that of more sophisticated regression models. ${ }^{11}$ The availability of their individual components and the simple scoring system promote their broad clinical application. Briefly , ESRS allocates one point each for the risk factors age 6575 years, hypertension, diabetes mellitus, active smoking, history of myocardial infarction, other cardiovascular disease, peripheral artery disease or cerebral ischaemic event prior to the index event and two points for age $>75$ years. SPI- 2 allocates one point for hypertension or coronary heart disease, two points for an index event of stroke (vs TIA) or age $>70$ years, and three points for diabetes, a prior history of stroke or heart failure. An ESRS $>2$ is considered high risk, while an SPI-2 score of 0-3 points is considered low, 4-7 intermediate and $8-15$ high risk.

Continuous measurements are given as means $\pm \mathrm{SD}$, unless otherwise stated, and categorical data as absolute count (percent). hsTropT values below the sensitivity threshold $(3 \mathrm{pg} / \mathrm{ml})$ were replaced by $1.5 \mathrm{pg} / \mathrm{ml}$. Group comparisons of continuous variables were performed using $t$ tests for independent samples and MannWhitney U tests. Proportions were compared using $\chi^{2}$ tests and Armitage's trend test where appropriate.

The occurrence of mortality and vascular events was depicted using Kaplan-Meier survival curves. HRs were estimated by Cox proportional hazard models and risk scores were calculated with estimated coefficients using $\exp \{-\operatorname{sum}(\mathrm{X} \times \beta)\}$. The concordance index (c-statistic) ${ }^{30}$ calculated by R-package survcomp measures the predictive performance of ESRS, SPI-II and hsTropT and their combinations. Risk estimation by ESRS or SPI-2 reflects recommended clinical practice and we therefore considered increasing the incremental predictive value of these models as clinically relevant. To investigate the additive predictive value of hsTropT in combination with ESRS, SPI-II or NIH-SS (as the most strongly associated indicator of stroke severity in our cohort), we fitted $\lg (\mathrm{hs}$ TropT) to base models constructed for each individual score and estimated improvement of the base model by $-2 \log$ (likelihood). Similarly, we constructed a base model for $\lg ($ hsTropT) and added each clinical score individually to assess how much prediction by hsTropT would be improved by the addition of the clinical scores. Estimated predicted probabilities allowed classification in discrete risk groups. Incremental discriminatory ability was further assessed by an integrated discrimination index (IDI) ${ }^{31}$ using the above-mentioned risk categories. Due to a non-normal distribution, marker values were log-transformed before being entered into generalised linear models.

All statistical analyses were performed with IBM SPSS Statistics V.18 and V.19 unless otherwise stated.

\section{RESULTS}

Two hundred and eighty-one patients were included in Find-AF. One withdrew informed consent. Four were lost to follow-up. In eight patients the final diagnosis for the index event was other than cerebral ischaemia. Seventy-one patients were excluded from the present analysis due to documented atrial fibrillation on baseline ECG $(n=42)$ or 7 -day Holter ECG $(n=29$, one of 
Table 1 Clinical characteristics

\begin{tabular}{|c|c|c|c|c|}
\hline & Overall cohort & hs-Troponin $\mathrm{T}<6.15 \mathrm{pg} / \mathrm{ml}(\mathrm{n}=98)$ & hs-Troponin $T \geq 6.15 \mathrm{pg} / \mathrm{ml}(\mathrm{n}=99)$ & $p$ Value \\
\hline \multicolumn{5}{|l|}{ Endpoints } \\
\hline All-cause death & 13 & 2 & 11 & 0.010 \\
\hline Vascular events & 23 & 5 & 18 & 0.004 \\
\hline Cardiovascular death & 6 & 1 & 5 & 0.053 \\
\hline Acute coronary syndrome & 6 & 2 & 4 & 0.145 \\
\hline Stroke & 12 & 3 & 9 & 0.077 \\
\hline \multicolumn{5}{|l|}{ Clinical characteristics } \\
\hline Female gender & $81(41.1 \%)$ & $43(43.9 \%)$ & $38(38.4 \%)$ & 0.433 \\
\hline Age (years) & $67 \pm 13$ & $62 \pm 13$ & $72 \pm 11$ & $<0.0005$ \\
\hline Body mass index $\left(\mathrm{kg} / \mathrm{m}^{2}\right)$ & $27.8 \pm 5.9$ & $27.6 \pm 5.6$ & $28.1 \pm 6.3$ & 0.568 \\
\hline $\mathrm{SBP}(\mathrm{mm} \mathrm{Hg})$ & $143 \pm 22$ & $142 \pm 21$ & $144 \pm 23$ & 0.478 \\
\hline $\mathrm{DBP}(\mathrm{mm} \mathrm{Hg})$ & $79 \pm 13$ & $80 \pm 11$ & $78 \pm 14$ & 0.333 \\
\hline Heart rate $(\mathrm{bpm})$ & $74 \pm 15$ & $71 \pm 12$ & $77 \pm 17$ & 0.009 \\
\hline Body temperature $\left({ }^{\circ} \mathrm{C}\right)$ & $36.8 \pm 0.4$ & $35.7 \pm 0.3$ & $36.8 \pm 0.4$ & 0.861 \\
\hline Previous stroke & $32(16.2 \%)$ & $14(14.3 \%)$ & $18(18.2 \%)$ & 0.459 \\
\hline Previous TIA & $19(9.6 \%)$ & $7(7.1 \%)$ & $12(12.1 \%)$ & 0.237 \\
\hline Heart failure & $10(5.1 \%)$ & $3(3.1 \%)$ & $7(7.1 \%)$ & 0.200 \\
\hline Hypertension & $140(71.1 \%)$ & $61(62.2 \%)$ & $79(79.8 \%)$ & 0.007 \\
\hline Diabetes mellitus & $43(21.8 \%)$ & $18(18.4 \%)$ & $25(25.3 \%)$ & 0.242 \\
\hline Smoker & $50(25.4 \%)$ & $33(33.7 \%)$ & $17(17.2 \%)$ & 0.024 \\
\hline Hyperlipidemia & $64(32.5 \%)$ & $35(35.7 \%)$ & $29(29.3 \%)$ & 0.336 \\
\hline Coronary artery disease & $23(11.7 \%)$ & $10(10.2 \%)$ & $13(13.1 \%)$ & 0.522 \\
\hline $\mathrm{T}_{\text {symptom onset }}(\mathrm{h})(\mathrm{IQR})$ & $4.4(2.0-10.5)$ & $4.5(2.0-11.5)$ & $4.4(2.0-9.5)$ & 0.465 \\
\hline NIH-SS (IQR) & $2(1-4)$ & $2(1-3)$ & $3(1-6)$ & $<0.0005$ \\
\hline Modified Rankin Scale score (IQR) & $2(1-3)$ & $2(1-3)$ & $2(1-4)$ & 0.001 \\
\hline ESRS (IQR) & $3(2-4)$ & $3(1-4)$ & $3(2-4)$ & 0.001 \\
\hline SPI-2 (IQR) & $5(3-6)$ & $3.5(2-6)$ & $5(2-7)$ & $<0.0005$ \\
\hline LV-EF (\%) & $62 \pm 9$ & $63 \pm 9$ & $61 \pm 10$ & 0.160 \\
\hline$E / e^{\prime}$ & $10.5 \pm 3.9$ & $9.9 \pm 3.5$ & $11.2 \pm 4.3$ & 0.024 \\
\hline \multicolumn{5}{|l|}{ Stroke classification } \\
\hline TIA & $68(34.5 \%)$ & $41(41.8 \%)$ & $27(27.3 \%)$ & \\
\hline Minor stroke & $55(27.9 \%)$ & $29(29.6 \%)$ & $26(26.3 \%)$ & \\
\hline Major stroke & $74(37.6 \%)$ & $28(28.6 \%)$ & $46(46.5 \%)$ & 0.007 \\
\hline \multicolumn{5}{|l|}{ TOAST classification } \\
\hline Large artery atherosclerosis & $39(19.8 \%)$ & $23(23.5 \%)$ & $16(16.2 \%)$ & \\
\hline Cardioembolic & $31(15.7 \%)$ & $11(11.2 \%)$ & $20(20.2 \%)$ & \\
\hline Lacunar/small vessels & $27(13.7 \%)$ & $9(9.2 \%)$ & $18(18.2 \%)$ & \\
\hline Rare/other causes & $5(2.5 \%)$ & $2(2.0 \%)$ & $3(3.0 \%)$ & \\
\hline Undetermined cause & $95(48.2 \%)$ & $53(54.1 \%)$ & $42(42.4 \%)$ & 1.000 \\
\hline \multicolumn{5}{|l|}{ Routine laboratory tests } \\
\hline Creatinine (mg/dl) & $0.97 \pm 0.53$ & $0.86 \pm 0.22$ & $1.09 \pm 0.69$ & 0.002 \\
\hline $\mathrm{CRP}(\mathrm{mg} / \mathrm{l})$ & $6.4 \pm 17.3$ & $4.5 \pm 8.0$ & $8.3 \pm 23.0$ & 0.126 \\
\hline Glucose $(\mathrm{mg} / \mathrm{dl})$ & $129 \pm 53$ & $120 \pm 36$ & $138 \pm 64$ & 0.020 \\
\hline Total cholesterol (mg/dl) & $196 \pm 46$ & $199 \pm 36$ & $194 \pm 54$ & 0.418 \\
\hline LDL cholesterol (mg/dl) & $130 \pm 39$ & $131 \pm 32$ & $128 \pm 45$ & 0.686 \\
\hline HDL cholesterol (mg/dl) & $51 \pm 13$ & $53 \pm 13$ & $49 \pm 14$ & 0.046 \\
\hline Triglycerides (mg/dl) & $131 \pm 67$ & $127 \pm 67$ & $136 \pm 67$ & 0.329 \\
\hline HbA1c (\%) & $6.2 \pm 1.1$ & $6.1 \pm 1.0$ & $6.3 \pm 1.2$ & 0.093 \\
\hline
\end{tabular}

whom had a previous history of paroxysmal atrial fibrillation). Among the analysis population of 197 patients, 148 (75.1\%) attended the clinical 1-year follow-up, 45 (22.8\%) underwent telephone follow-up (including relatives of nine patients who had died between 3 months and 1 year), and four (2.0\%) had died before the 90-day follow-up. Baseline clinical characteristics are shown in table 1 .
After a median follow-up of 368 (IQR 358-378) days, there were 23 vascular events as defined above. Thirteen patients died, six from cardiovascular causes. As one patient died from stroke, the sum of the individual components of the vascular endpoint listed in table 1 is 24 .

hsTropT and GDF-15 were significantly elevated in patients with an adverse vascular 1-year outcome. In univariate Cox 
A

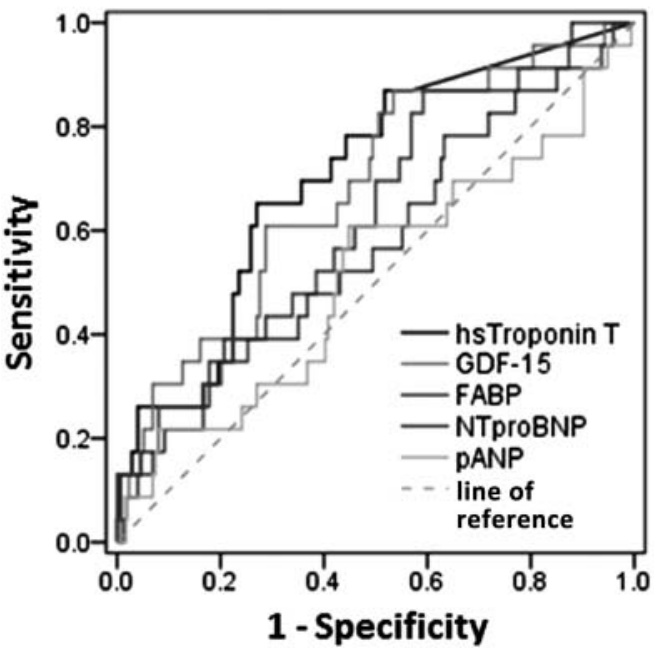

B

Overall mortality

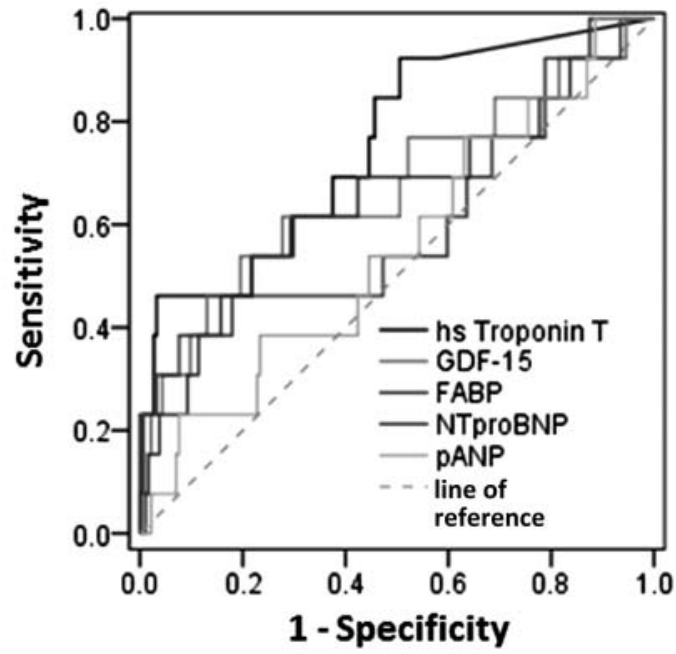

Figure 1 Receiver operating characteristic curves for individual markers to predict (A) vascular events or (B) overall mortality. hsTroponin $\mathrm{T}_{\text {, }}$ high-sensitivity troponin T. FABP, fatty acid binding protein; GDF-15, growth differentiation factor 15; hsTroponin T, high-sensitivity troponin T; NTproBNP, N-terminal pro brain natriuretic peptide; pANP, pro atrial natriuretic peptide.

regression, hsTropT $(\mathrm{p}<0.001)$, h-FABP $\quad(\mathrm{p}=0.023)$ and GDF-15 $(p=0.009)$ were predictive of an adverse vascular 1-year outcome, while hsTropT $(\mathrm{p}<0.001)$, GDF-15 $(\mathrm{p}=0.007)$ and NT-proBNP $(p=0.050)$ were associated with total mortality (an association with h-FABP was barely insignificant: $\mathrm{p}=0.051$ ) (receiver operating characteristic (ROC) curves for individual markers are shown in figure 1). When all markers were combined in multivariate Cox regression, only hsTropT was independently predictive for both vascular outcome $(p=0.045)$ and total mortality $(p=0.004)$. We focused on hsTropT as an individual marker because it was by far the most strongly predictive in univariate analysis.

hsTropT was measurable above the limit of detection in 115 (59.9\%) patients, and 48 (24.4\%) had an hsTropT level above the upper limit of normal (ULN) of $14 \mathrm{pg} / \mathrm{ml}$. The median value was 6.15 (IQR 3.00-13.9) pg / ml. All endpoints occurred more frequently in the group with hsTropT above the median (table 1).

\section{Vascular risk}

The HR for the time to a first vascular event was 3.86 (95\% CI 1.43-10.4) $(p=0.008$; figure 1A) for patients with hsTropT above versus below the median. When dichotomised according to the ULN, the HR was 2.20 (95\% CI 0.949-5.07) ( $p=0.066$; figure 1A). When clinical scores ESRS, SPI-2 or NIH-SS were added as a covariable to a Cox regression model based on $\mathrm{lg}$ (hsTropT), only SPI-2 significantly improved the predictive model for vascular events (table 2A). Accordingly, when base models were built from each of the clinical scores, the inclusion of $\lg ($ hs $\operatorname{Trop} \mathrm{T})$ as covariable strongly and significantly improved prediction (table $2 \mathrm{~B}$ ).

The c-statistic to predict vascular events was $0.710(0.608-$ $0.811)$ for hsTropT, $0.695(0.567-0.822)$ for ESRS and 0.699 $(0.587-0.810)$ for SPI-2. When clinical scores were combined with hsTropT, the c-statistic improved to 0.747 (0.651-0.843) $(\mathrm{p}=\mathrm{NS})$ for ESRS plus hsTropT and to $0.763(0.672-0.855)$ $(\mathrm{p}=\mathrm{NS}$ ) for SPI-2 plus hsTropT (see the ROC curves in figure $3 \mathrm{~A})$. As regards reclassification, IDI was $5.5 \% \quad(p=0.237)$ when hsTropT was added to ESRS and 21.9\% $(\mathrm{p}=0.006)$ when hsTropT was added to SPI-2. Not a single patient in the low-risk category of ESRS $(\leq 2)$ and with hsTropT below the median and only two patients $(3.3 \%)$ with ESRS $\leq 2$ and hsTropT $<14 \mathrm{pg} / \mathrm{ml}$ experienced a vascular event during follow-up (table 3 ). Results were similar when we included the patients who had been diagnosed with atrial fibrillation at baseline, with significant improvement in predictive models based on the clinical scores, albeit the HR for patients with an elevated hsTropT tended to be lower (HR 2.64 for patients above versus below the median, $\mathrm{p}=0.028)$ and the $\mathrm{c}$-statistic was lower $(0.670 ; 0.569-0.771)$.

\section{All-cause mortality}

The HR for all-cause mortality for patients with hsTropT above versus below the median was $5.95(1.32-26.9) \quad(p=0.020$ log-rank test; figure $1 \mathrm{~B})$ and $3.82(1.28-11.4)$ with hsTropT above versus below $14 \mathrm{pg} / \mathrm{ml}(\mathrm{p}=0.016$; figure $1 \mathrm{~B}) . \lg$ (hsTropT) strongly and significantly improved Cox regressional predictive models based on clinical scores for all-cause mortality (table 2B), while a predictive model based on $\lg (\mathrm{hs}$ Trop $\mathrm{T}$ ) was slightly improved by SPI-2 and NIH-SS only (table 2A). The c-statistic improved from $0.744(0.575-0.912)$ for ESRS to 0.793 (0.669$0.918)(\mathrm{p}=\mathrm{NS})$ and from $0.708(0.549-0.867)$ for SPI-2 to $0.795(0.656-0.934)(p=N S)$ for the respective combinations with hsTropT. The c-statistic for hsTropT alone was 0.751 (0.624-0.878) (see the ROC curves in figure 3B).

IDI was $9.4 \%(p=0.102)$ when hsTropT was added to ESRS and $31.3 \%(\mathrm{p}<0.001)$ when hsTropT was added to SPI-2. Again, no patient with hsTropT below the median and ESRS $\leq 2$ and only one patient with ESRS $\leq 2$ and hsTropT $<14 \mathrm{pg} / \mathrm{ml}$ died during follow-up. As for vascular events, the predictive power of hsTropT was slightly lower when we included patients with atrial fibrillation (HR 3.46, p $=0.057$, for hsTropT above versus below the median, prediction by scores all significantly improved by hsTropT).

\section{DISCUSSION}

We find that in patients with cerebral ischaemia, hsTropT is elevated in those with future vascular events or death, and that it is predictive for these events beyond established clinical risk 
Table 2 Cox regression models to predict vascular events or total mortality

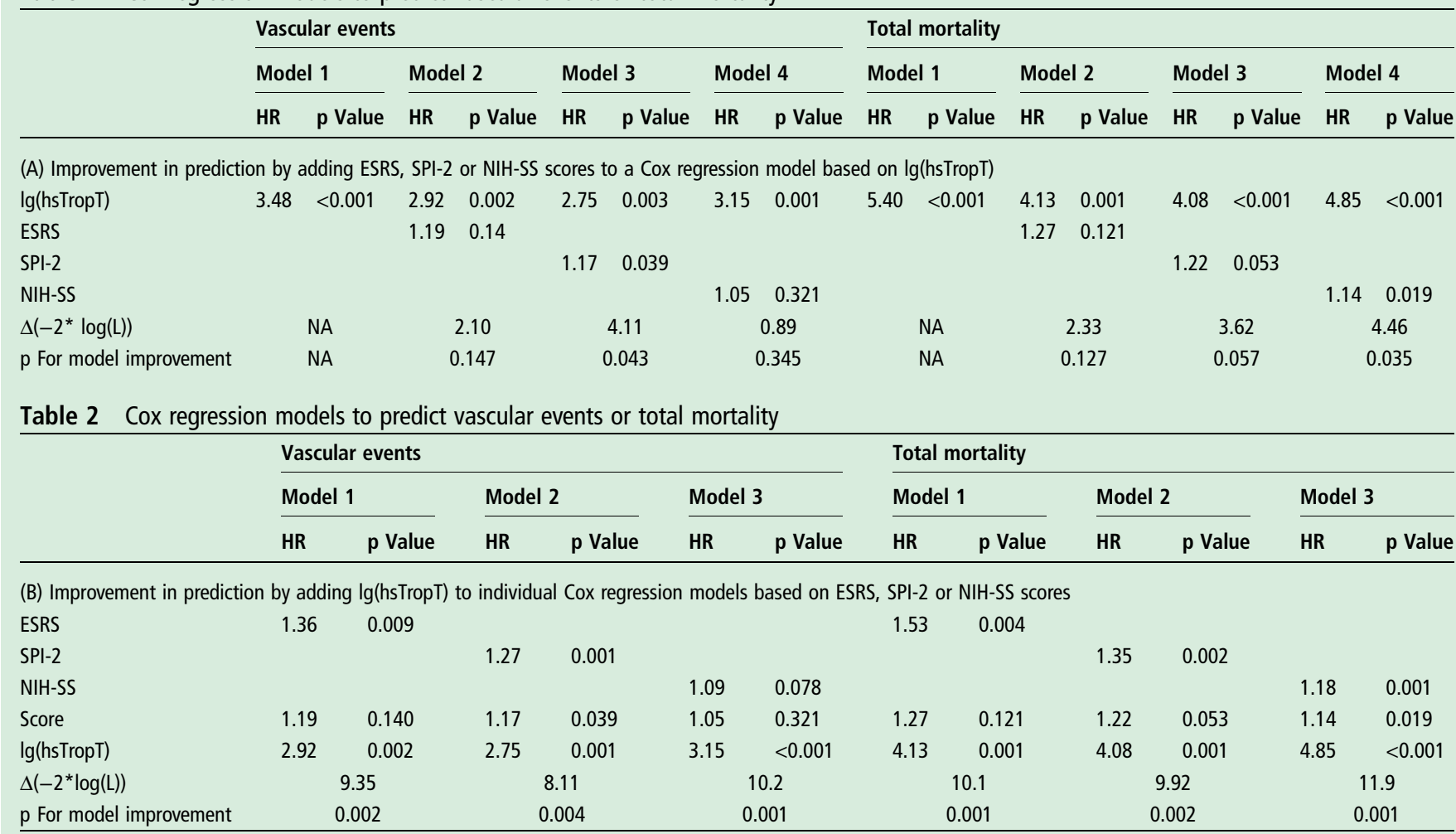

HRs are given per unit increase in the respective variable.

ESRS, Essen Stroke Risk Score; hsTropT, high-sensitivity troponin T; NIH-SS, National Institutes of Health Stroke Scale; SPI-2, Stroke Prognosis Instrument 2.

scores. This single biomarker appears to carry prognostic information comparable to that of clinical risk scores composed of several variables.

\section{Prognostic biomarkers in cerebral ischaemia and methodological considerations}

Numerous potentially prognostic markers have been investigated in patients with cerebral ischaemia with varying success. ${ }^{13} 23$ 32-34 Many of the studies had small sample sizes and limited follow-up, ${ }^{13}$ focused on all-cause mortality and functional outcome rather than cardiovascular risk, and did not correct for established clinical risk scores. Recently, however, large welldesigned studies have reported more novel cardiovascular markers to be predictive for all-cause mortality and functional outcome in stroke patients. ${ }^{21} 23{ }^{32-34}$ Although undeniably the most important outcome for the patient, all-cause mortality is not disease-specific and the translation of an increased risk into specific preventive measures may be challenging as a result. We therefore chose pathophysiologically linked vascular events as the combined primary endpoint for our analysis. An increased risk for vascular events may be targeted by anti-atherothrombotic therapies such as antiplatelet administration, lipid-lowering drugs or lifestyle modification. In comparison with previous studies, several factors make our analysis methodologically suitable for identifying a new biomarker, for example, marker measurement blinded to clinical variables and outcome, consecutive enrolment of unselected cases, multivariate analysis with the incorporation of established clinical risk scores, a defined enrolment period, prospective definition and assessment of data and all endpoints, comparison of five novel cardiovascular biomarkers in order to select and benchmark that with the strongest predictive value, and the reporting of reclassification indices. ${ }^{35}$ We also assessed atrial fibrillation using a highly sensitive diagnostic method ${ }^{19}$ and excluded affected patients (who need a different secondary prevention strategy) from our analysis which focused on atherosclerotically driven cardiovascular prognosis. In fact this may explain the lack of prognostic value shown by NT-proBNP in our study, contrasting with a recent multimarker analysis $^{21}$ that may have included patients with elevated NT-proBNP due to undiagnosed atrial fibrillation (by itself limiting prognosis). As highly sensitive methods for the detection of atrial fibrillation may not be used in current clinical practice, we repeated our analyses for all patients including those with atrial fibrillation, and found largely similar results.

With regard to the prediction of vascular events instead of general functional outcome, the literature on biomarker approaches is limited for stroke patients, although the ESRS and SPI-2 both show similar accuracy in predicting stroke recurrence compared to other vascular events. ${ }^{71}$ As we targeted cardiovascular pathology, we limited our analysis to cardiovascular markers representing different pathophysiological domains. Although several markers were predictive for an adverse outcome in univariate analysis, hsTropT was the strongest predictor for both vascular events and all-cause mortality and the only marker that remained significantly predictive in multivariate analysis. We acknowledge, however, that our analysis is underpowered to unravel smaller increments in prediction achieved with marker combinations or comparisons with established markers such as D-dimer, CRP or fibrinogen. ${ }^{14} 15$ GDF-15, for example, was elevated in patients with an adverse outcome, was predictive for functional outcome after 90 days in 


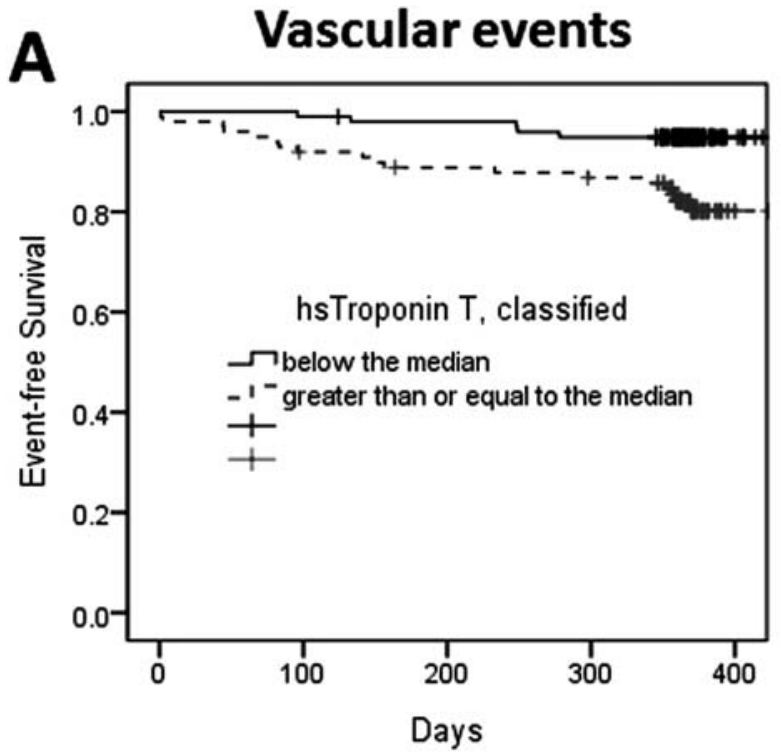

B Overall mortality

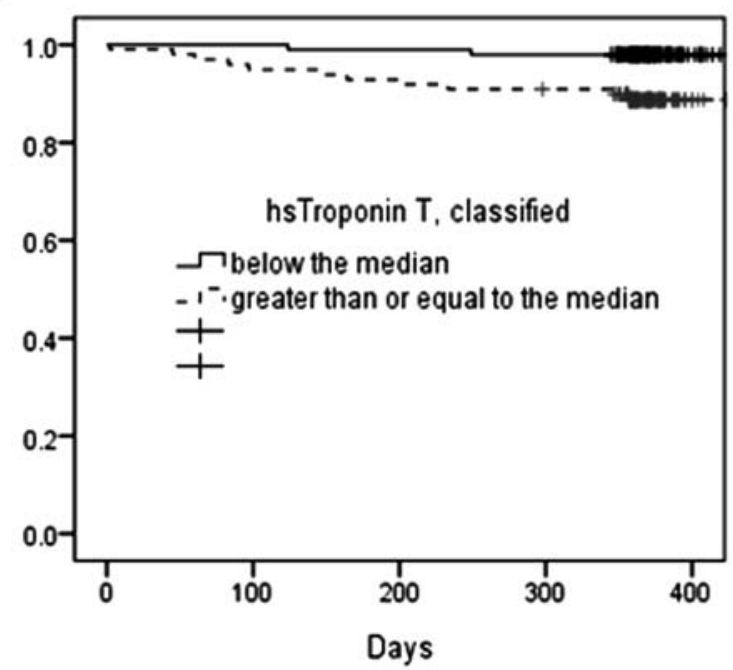

\section{Vascular events}

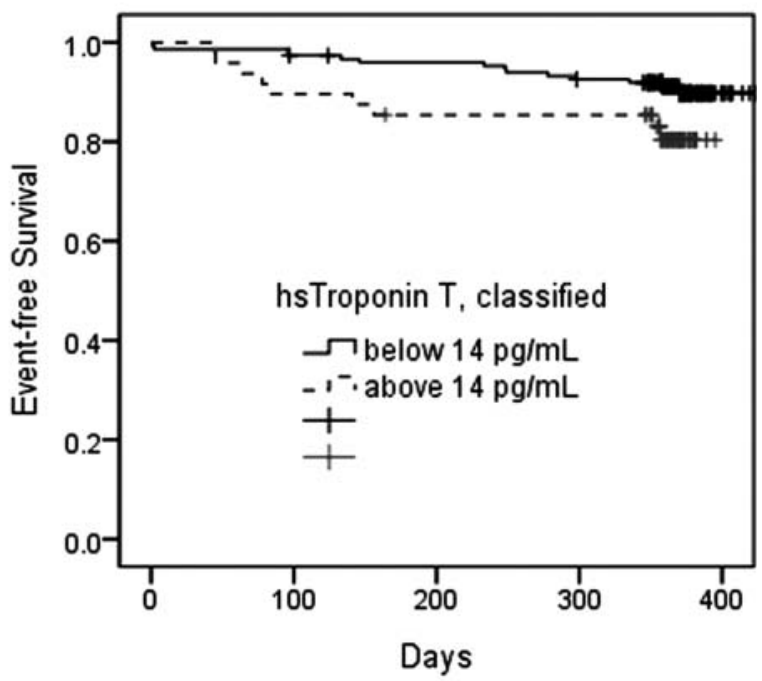

Overall mortality

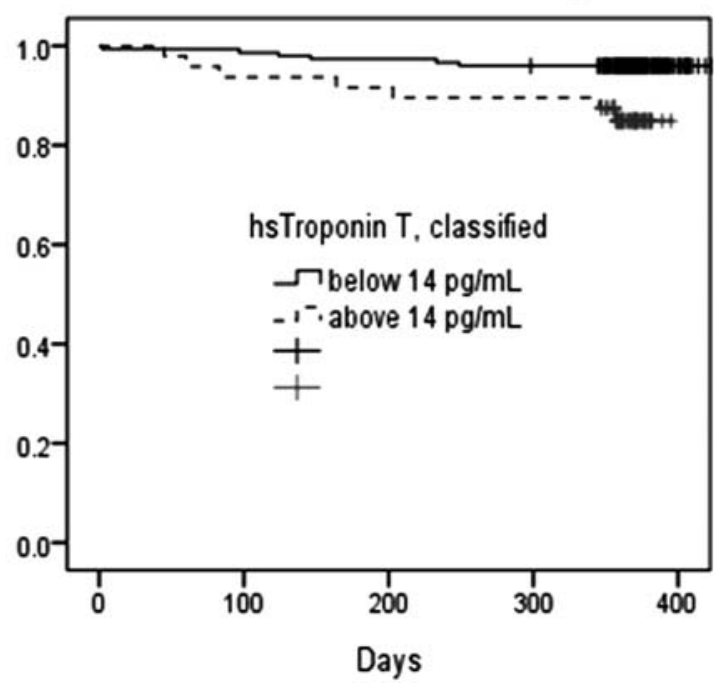

Figure 2 Kaplan-Meier plots for (A) survival free from vascular events and (B) overall survival according to high-sensitivity troponin T above or below the median and above or below the upper limit of normal.

another cohort ${ }^{36}$ as well as in our own, ${ }^{37}$ and might well have been found to be incrementally predictive in a larger cohort with a greater number of events .

Troponins for outcome in patients with cerebral ischaemia Before the advent of highly sensitive troponin assays, an association between troponin elevation and mortality was repeatedly reported (reviewed in Kerr et $a l^{38}$ ), although the independent predictive value of troponin was addressed in two studies only and none investigated reclassification. Our observation that hsTropT is independently predictive in patients with cerebral ischaemia confirms these reports by using a highly sensitive assay that allowed for the detection and quantification of troponin values in the normal range in a large proportion of the study sample. The approximately $25 \%$ of patients with troponin values above the ULN is well in line with older reports on standard troponin assays. The mechanism of troponin elevation in acute stroke patients has been debated but remains unclear. ${ }^{38}$ One explanation could be the coexistence of acute cerebral ischaemia with cardiac ischaemia and infarction. In fact, ischaemic changes shown on ECG are found more often in stroke patients with elevated troponin than in those without. ${ }^{38}$ In this sense, some of our patients might actually have undergone non-ST elevation myocardial infarction at the time of presentation. However, because marker values were determined post hoc, we cannot secure or rule out a diagnosis of myocardial infarction. It should be noted in this context that nearly all the outcome events occurred more than 2 weeks after presentation and only one fourth were ACSs. This argues against the otherwise tempting speculation that hsTropT was predictive for vascular events because patients already had an ACS on presentation that was missed at that time and diagnosed after some delay. As a second mechanism, raised troponin values have been described as due to elevated sympathoadrenal activation after insular strokes with consecutive patchy cardiomyocyte necrosis. ${ }^{39}$ In this sense, troponin would be a surrogate for insular strokes, but it remains unclear why it should then be predictive for future vascular events. More likely explanations for troponin elevation after cerebral ischaemia and its association with future vascular events are, on the one hand, subtle myocardial ischaemia at 
Figure 3 Receiver operating characteristic curves to predict (A) vascular events or (B) overall mortality for hsTropT, clinical risk scores Essen Stroke Risk Score (ESRS) and Stroke Prognosis Instrument 2 (SPI-2), or the combination of scores with highsensitivity troponin $\mathrm{T}$ (hsTroponin T).

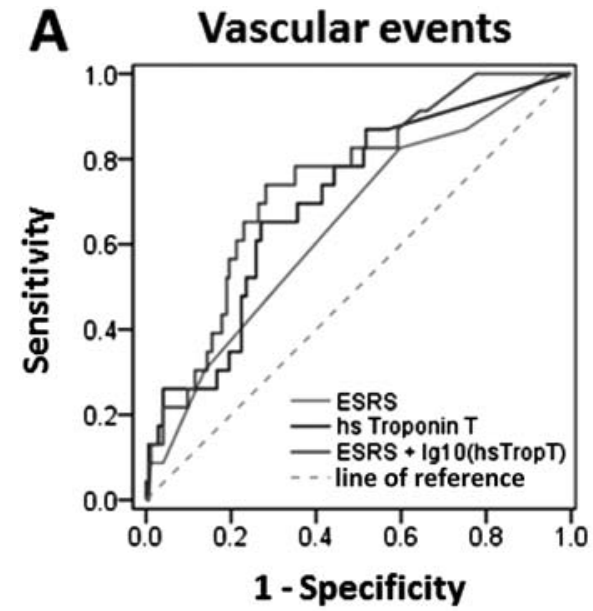

B Overall mortality

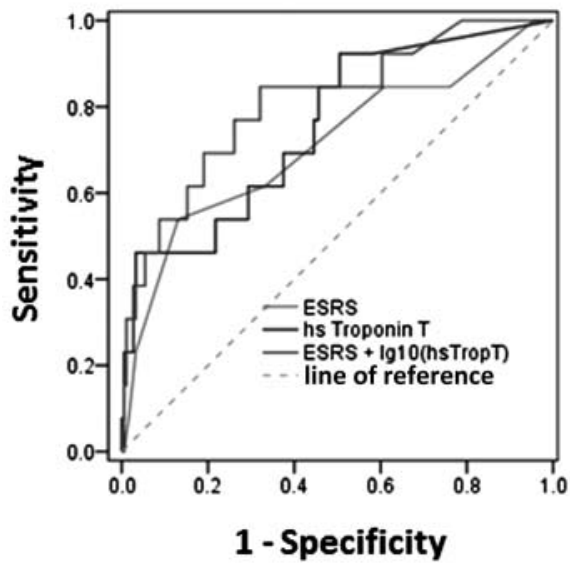

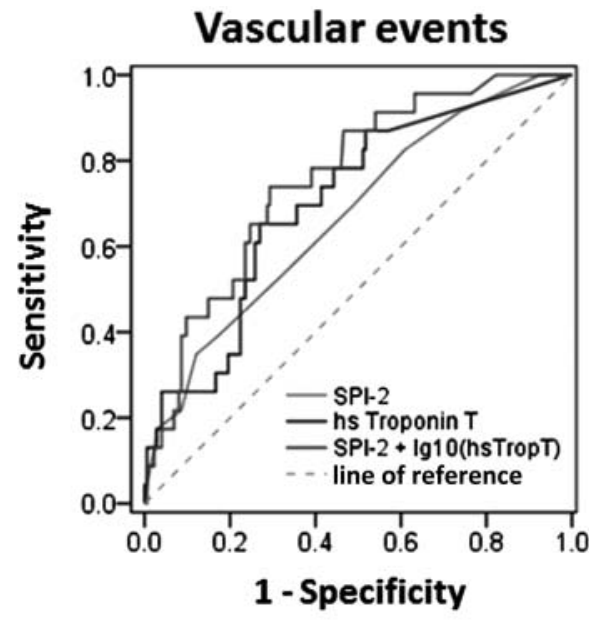

Overall mortality

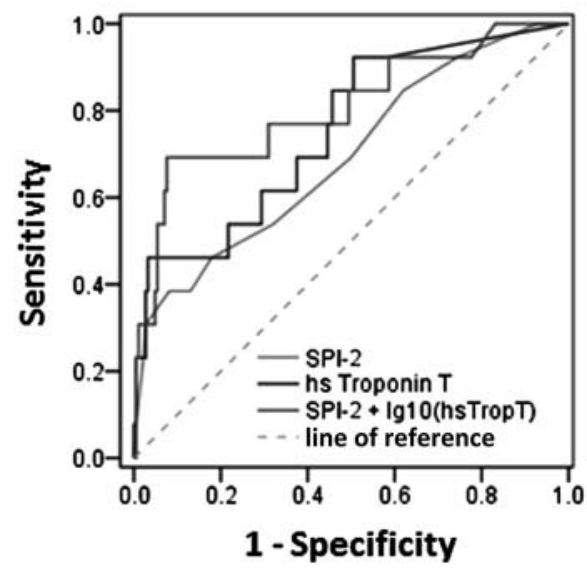

presentation due to acute haemodynamic and autonomic nervous derangements at the time of stroke. These perturbances might, if severe enough, lead to troponin release by themselves or, in certain patients, in combination with pre-existing, albeit previously subclinical, cardiac disease (mainly coronary artery disease and heart failure). Secondly, troponin levels in the normal range are associated with cardiovascular risk, even without elevations beyond the ULN. ${ }^{40}$ These measurable troponin levels are likely a result of cardiomyocyte necrosis and apoptosis $^{41}$ and may therefore be a sensitive marker of cardiac

\begin{tabular}{llll}
$\begin{array}{l}\text { Table } 3 \\
\text { hsTropT }\end{array}$ & Vascular events according to ESRS risk category and \\
\hline \multicolumn{5}{c}{} & hsTropT according to median \\
\cline { 2 - 4 } & hsTropT $<6.15 \mathrm{pg} / \mathrm{ml}$ & hsTropT $\geq 6.15 \mathrm{pg} / \mathrm{ml}$ & Total \\
\hline ESRS $\leq 2$ & $0 / 47(0 \%)$ & $4 / 27(14.8 \%)$ & $4 / 74(5.4 \%)$ \\
ESRS $>2$ & $5 / 53(9.4 \%)$ & $14 / 70(20.0 \%)$ & $19 / 123(15.4 \%)$ \\
Total & $5 / 100(5.0 \%)$ & $18 / 97(18.6 \%)$ & $23 / 197(11.7 \%)$ \\
\hline \multicolumn{5}{c}{ hsTropT according to ULN } \\
\cline { 2 - 4 } & hsTropT $<14 \mathrm{pg} / \mathrm{ml}$ & hsTropT $\geq 14 \mathrm{pg} / \mathrm{ml}$ & Total \\
\hline ESRS $\leq 2$ & $2 / 60(3.3 \%)$ & $2 / 14(14.3 \%)$ & $4 / 74(5.4 \%)$ \\
ESRS $>2$ & $12 / 89(13.5 \%)$ & $7 / 34(20.6 \%)$ & $19 / 123(15.4 \%)$ \\
Total & $14 / 149(9.4 \%)$ & $9 / 48(18.8 \%)$ & $23 / 197(11.7 \%)$ \\
\hline
\end{tabular}

ESRS, Essen Stroke Risk Score; hsTropT, high-sensitivity troponin T; ULN, upper limit of normal. physiology and potentially result from different subtle clinical derangements that stress the heart but are otherwise difficult to quantify due to their heterogeneous nature. Such a function is supported by the observation of an association between troponin levels in the normal range and different cardiovascular risk factors and subclinical atherosclerotic disease. ${ }^{42}$ Further insight into the link between troponin elevation and recurrent events might be gained by comparing the predictive power of troponin levels between different aetiological subgroups (eg, according to the TOAST classification). The numbers in our cohort, however, are too small for such an analysis. Whatever the reasons for troponin elevation in stroke patients, the mechanisms described suggest a biological rationale for our observation that hsTropT levels have a prognostic value in stroke patients.

\section{Clinical relevance and perspective}

Although clinical risk prediction scores have been repeatedly validated and are therefore established prognostic tools for patient management in cerebral ischaemia, their predictive value is only moderate. ${ }^{11}$ Commercially available biomarkers like hsTropT are highly standardised and reproducible surrogates for biological processes that are extremely objective and easy to interpret when physicians are provided with clear rules on their use. Due to their high degree of standardisation, they allow for comparisons across different settings (eg, different countries or trials). We therefore believe that our data on the prognostic value of hsTropT levels in patients with cerebral ischaemia indicate a future role for this biomarker as a prognostic tool for both clinicians and clinical researchers. 


\section{Limitations}

While our cohort is large enough to reveal the statistically significant predictive value of hsTropT for cardiovascular risk assessment in patients with cerebral ischaemia, it is too small to derive clear cut recommendations on the optimal use of hsTropT in risk assessment. Consequently, validation in a large cohort of patients with cerebral ischaemia is urgently needed and should be a high priority future project. The differences in most measures of reclassification, although numerically uniformly favouring hsTropT, were not significant. Our pilot analysis is limited by sample size and event rate in our cohort, which was designed for a different purpose. Furthermore, the present analysis was not predefined and was performed post hoc. Prospective validation of our results in a large-scale cohort will therefore be crucial. Of note, c-statistics were not only consistently numerically improved when hsTropT was combined with ESRS or SPI-2, but were also all numerically higher for hsTropT alone, as compared to ESRS or SPI-2 alone.

\section{SUMMARY}

In summary, we show that hsTropT has predictive value for future vascular events beyond established clinical models in acute cerebral ischaemia. Our data warrant validation in larger cohorts to establish the optimal method of integrating hsTropT levels into risk assessment. Better risk prediction will lead to an improvement in risk-tailored secondary preventive measures and ultimately in outcomes for these patients.

Acknowledgements This study was supported by an unrestricted minor grant from Roche Diagnostics. Biomarkers were measured at Roche Diagnostics Laboratories (see Methods section above)

Contributions RS, KG and RW designed the trial, cleaned and analysed the data, and drafted and revised the paper. All three are guarantors. CN collected data and contributed significantly to analysing the data. FE collected data and critically reviewed the manuscript. MM performed the statistical analysis and reviewed the manuscript. JW, $\mathrm{KW}$ and JS contributed significantly to data collection and analysis as well as critical review of the manuscript. GH took part in trial design and reviewed the manuscript.

Competing interests None.

Ethics approval The Ethics Committee of the University of Göttingen approved this study.

Provenance and peer review Not commissioned; externally peer reviewed

Open Access This is an Open Access article distributed in accordance with the Creative Commons Attribution Non Commercial (CC BY-NC 3.0) license, which permits others to distribute, remix, adapt, build upon this work non-commercially, and license their derivative works on different terms, provided the original work is properly cited and the use is non-commercial. See: http://creativecommons.org/licenses/by-nc/3.0/

\section{REFERENCES}

1 Hardie K, Hankey GJ, Jamrozik K, et al. Ten-year risk of first recurrent stroke and disability after first-ever stroke in the Perth Community Stroke Study. Stroke 2004;35:731-5.

2 Steg PG, Bhatt DL, Wilson PW, et al. One-year cardiovascular event rates in outpatients with atherothrombosis. JAMA 2007;297:1197-206.

3 Touze E, Varenne 0 , Chatellier G, et al. Risk of myocardial infarction and vascular death after transient ischemic attack and ischemic stroke: a systematic review and meta-analysis. Stroke 2005;36:2748-55

4 CAPRIE Steering Committee. A randomised, blinded, trial of clopidogrel versus aspirin in patients at risk of ischaemic events (CAPRIE). Lancet 1996;348:1329-39.

5 Diener HC, Ringleb PA, Savi P. Clopidogrel for the secondary prevention of stroke. Expert Opin Pharmacother 2005;6:755-64.

6 Viscoli CM, Brass LM, Kernan WN, et al. A clinical trial of estrogen-replacement therapy after ischemic stroke. N Engl J Med 2001;345:1243-9.

7 Weimar C, Diener HC, Alberts MJ, et al. The Essen stroke risk score predicts recurrent cardiovascular events: a validation within the REduction of Atherothrombosis for Continued Health (REACH) registry. Stroke 2009;40:350-4

8 Weimar C, Goertler M, Rother J, et al. Predictive value of the Essen Stroke Risk Score and Ankle Brachial Index in acute ischaemic stroke patients from 85 German stroke units. J Neurol Neurosurg Psychiatry 2008;79:1339-43.
9 Weimar C, Goertler M, Rother J, et al. Systemic risk score evaluation in ischemic stroke patients (SCALA): a prospective cross sectional study in 85 German stroke units. J Neurol 2007:254:1562-8.

10 Kernan WN, Viscoli CM, Brass LM, et al. The stroke prognosis instrument II (SPI-II): a clinical prediction instrument for patients with transient ischemia and nondisabling ischemic stroke. Stroke 2000;31:456-62.

11 Weimar C, Benemann J, Michalski D, et al. Prediction of recurrent stroke and vascular death in patients with transient ischemic attack or nondisabling stroke: a prospective comparison of validated prognostic scores. Stroke 2010;41: 487-93.

12 Diener HC. Primär- und Sekundärprävention der zerebrale Ischämie. In: Diener HC ed. Leitlinien für Diagnostik und Therapie in der Neurologie. Stuttgart: Georg Thieme Verlag, 2008:654 ff.

13 Whiteley W, Chong WL, Sengupta A, et al. Blood markers for the prognosis of ischemic stroke: a systematic review. Stroke 2009;40:e380-9.

14 Whiteley W, Jackson C, Lewis S, et al. Association of circulating inflammatory markers with recurrent vascular events after stroke: a prospective cohort study. Stroke 2011:42:10-16

15 Welsh $\mathrm{P}$, Barber M, Langhorne $\mathrm{P}$, et al. Associations of inflammatory and haemostatic biomarkers with poor outcome in acute ischaemic stroke. Cerebrovasc Dis 2009;27:247-53.

16 Velagaleti RS, Gona P, Larson MG, et al. Multimarker approach for the prediction of heart failure incidence in the community. Circulation 2010;122:1700-6.

17 Schnabel RB, Schulz A, Messow CM, et al. Multiple marker approach to risk stratification in patients with stable coronary artery disease. Eur Heart J 2010;31:3024-31.

18 Zethelius B, Berglund L, Sundstrom J, et al. Use of multiple biomarkers to improve the prediction of death from cardiovascular causes. $N$ Engl J Med 2008:358:2107-16.

19 Stahrenberg R, Weber-Kruger M, Seegers J, et al. Enhanced detection of paroxysma atrial fibrillation by early and prolonged continuous holter monitoring in patients with cerebral ischemia presenting in sinus rhythm. Stroke 2010;41:2884-8.

20 Stahrenberg R, Edelmann F, Haase B, et al. Transthoracic echocardiography to rule out paroxysmal atrial fibrillation as a cause of stroke or transient ischemic attack. Stroke 2011;42:3643-45.

21 Whiteley W, Wardlaw J, Dennis M, et al. The use of blood biomarkers to predict poor outcome after acute transient ischemic attack or ischemic stroke. Stroke 2012:43:86-91.

22 Farmakis D, Filippatos G, Tubaro M, et al. Natriuretic peptides in acute coronary syndromes: prognostic value and clinical implications. Congest Heart Fail 2008;14(4 Suppl 1):25-9.

23 Katan M, Fluri F, Schuetz $\mathrm{P}$, et al. Midregional pro-atrial natriuretic peptide and outcome in patients with acute ischemic stroke. J Am Coll Cardiol 2010;56:1045-53.

24 Khan SQ, Dhillon O, Kelly D, et al. Plasma N-terminal B-Type natriuretic peptide as an indicator of long-term survival after acute myocardial infarction: comparison with plasma midregional pro-atrial natriuretic peptide: the LAMP (Leicester Acute Myocardial Infarction Peptide) study. J Am Coll Cardio 2008:51:1857-64.

25 Kempf T, Sinning JM, Quint A, et al. Growth-differentiation factor-15 for risk stratification in patients with stable and unstable coronary heart disease: results from the AtheroGene study. Circulation. Cardiovasc Genet 2009;2:286-92.

26 Ivandic BT, Spanuth E, Kleber M, et al. High-sensitivity troponin T improves the prognostic value of $\mathrm{N}$-terminal pro-B-type natriuretic peptide in patients with stable coronary artery disease: results from the LURIC Study. Clin Chem Lab Med 2011:49:1053-8

27 Ndrepepa G, Braun S, Mehilli J, et al. Prognostic value of sensitive troponin T in patients with stable and unstable angina and undetectable conventional troponin. Am Heart J 2011;161:68-75.

28 Kilcullen N, Viswanathan K, Das R, et al. Heart-type fatty acid-binding protein predicts long-term mortality after acute coronary syndrome and identifies high-risk patients across the range of troponin values. J Am Coll Cardiol 2007;50:2061-7.

29 Klemt VJJ. Distribution of GDF-15 in apparently healthy individuals assessed with a pre-commercial assay based on the ECLIA principle. AACC: Atlanta, Georgia, USA, 2011.

30 Haibe-Kains B, Desmedt C, Sotiriou C, et al. A comparative study of survival models for breast cancer prognostication based on microarray data: does a single gene beat them all? Bioinformatics 2008;24:2200-8.

31 Pencina MJ, D'Agostino RB Sr, D'Agostino RB, et al. Jr. Evaluating the added predictive ability of a new marker: from area under the ROC curve to reclassification and beyond. Stat Med 2008;27:157-72; discussion 207-12.

32 Urwyler SA, Schuetz P, Fluri F, et al. Prognostic value of copeptin: one-year outcome in patients with acute stroke. Stroke 2010;41:1564-7.

33 Katan M, Fluri F, Morgenthaler NG, et al. Copeptin: a novel, independent prognostic marker in patients with ischemic stroke. Ann Neurol 2009;66:799-808.

34 Whiteley $W_{\text {, Jackson }}$, Lewis $S$, et al. Inflammatory markers and poor outcome after stroke: a prospective cohort study and systematic review of interleukin-6. PLoS Med 2009:6:e1000145. 
35 Hlatky MA, Greenland P, Arnett DK, et al. Criteria for evaluation of novel markers of cardiovascular risk: a scientific statement from the American Heart Association. Circulation 2009;119:2408-16.

36 Worthmann H, Kempf T, Widera C, et al. Growth differentiation factor 15 plasma levels and outcome after ischemic stroke. Cerebrovasc Dis 2011;32:72-8.

37 Groschel K, Schnaudigel S, Edelmann F, et al. Growth-differentiation factor-15 and functional outcome after acute ischemic stroke. J Neurol 2012:259:1574-9.

38 Kerr G, Ray G, Wu O, et al. Elevated troponin after stroke: a systematic review. Cerebrovasc Dis 2009:28:220-6.
39 Barber M, Morton JJ, Macfarlane PW, et al. Elevated troponin levels are associated with sympathoadrenal activation in acute ischaemic stroke. Cerebrovasc Dis 2007;23:260-6.

40 Zethelius B, Johnston N, Venge P. Troponin I as a predictor of coronary heart disease and mortality in 70-year-old men: a community-based cohort study. Circulation 2006:113:1071-8.

41 Giannitsis E, Katus HA. Troponins and high-sensitivity troponins as markers of necrosis in CAD and heart failure. Herz 2009;34:600-6.

42 Eggers KM, Lind L, Ahlstrom H, et al. Prevalence and pathophysiological mechanisms of elevated cardiac troponin I levels in a population-based sample of elderly subjects. Eur Heart J 2008;29:2252-8.

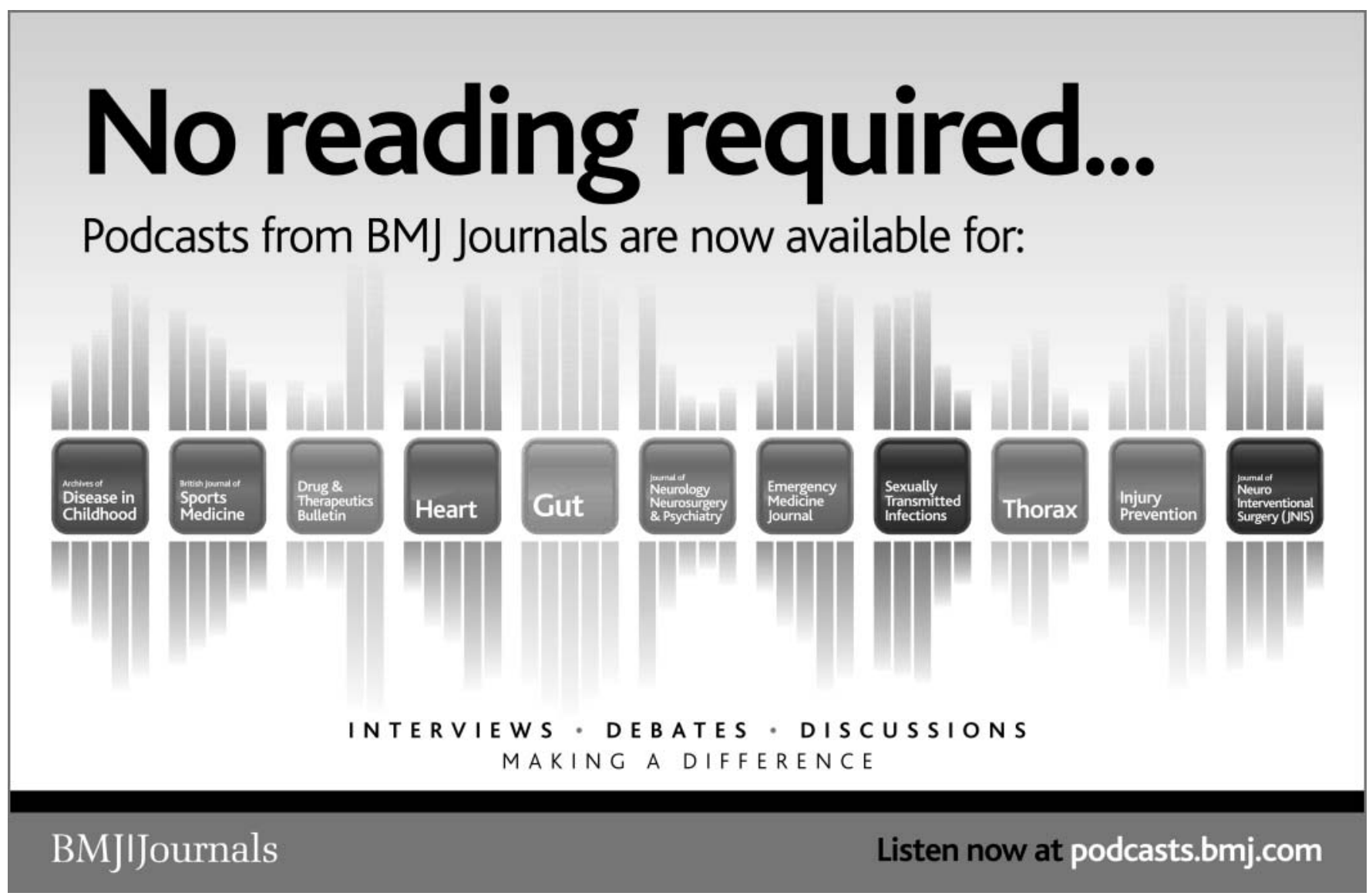

